INVESTIGACIÓN

https://doi.org/10.15198/seeci.2019.50.129-147

Recibido: 06/10/2019 --- Aceptado: 15/10/2019 --- Publicado: 15/11/2019

\title{
LAS PÁGINAS WEB DE LOS CENTROS EDUCATIVOS. ANÁLISIS DE LA SITUACIÓN ACTUAL EN LA COMUNIDAD VALENCIANA
}

\section{THE WEBSITES OF SCHOOLS. ANALYSIS OF THE CURRENT SITUATION IN THE VALENCIAN COMMUNITY}

\begin{abstract}
D 8 Juan Francisco Álvarez Herrero: Universidad de Alicante. España. juanfran.alvarez@ua.es
\end{abstract}

D 8 Rosabel Roig-Vila: Universidad de Alicante. España.

rosabel.roig@ua.es

\section{RESUMEN}

La página web se ha convertido en un requisito imprescindible de cualquier centro educativo. Más allá de ser plataformas para la información y la comunicación de toda la comunidad educativa, en la actualidad nos encontramos con que estas páginas web son también escaparates de los centros hacia la sociedad. En ellas, se anuncian y se muestran las excelencias de dichos centros y son un reclamo del márketing educativo. Por ello, se hace imprescindible conocer si estas "cartas" de presentación de los centros tienen todos los elementos y las características que deben poseer. Este estudio analizó 100 páginas web al azar de centros educativos no universitarios de la Comunidad Valenciana (España) y se constató que existen bastantes diferencias entre las webs de centros públicos y las de centros concertados y privados. En general, todas ellas presentaban alguna que otra deficiencia, entre las que destacan: una falta de imagen y una estética sugerente, falta de información, falta de actualización, el no uso ni difusión de las redes sociales del centro y la no difusión o información de las metodologías docentes o el estilo pedagógico del centro. Estos problemas vienen motivados por la falta de una correcta gestión y mantenimiento de las páginas web, función que muchas veces recae en los coordinadores TIC del centro y que pocas veces disponen de tiempo y formación para desempeñar dicha tarea. Así mismo, se detectó que las webs de estos centros educativos pocas veces tienen una finalidad pedagógica que incentive y promueva el aprendizaje.

PALABRAS CLAVE: páginas web de centro educativo - TIC - márketing educativo contenido web - Coordinador TIC - centros educativos - innovación.

${ }^{1}$ Juan Francisco Álvarez Herrero: Profesor asociado del Departamento de Didáctica General y Didácticas Específicas de la Facultad de Educación en la Universidad de Alicante (España). Doctor en Tecnología Educativa y Máster Universitario en Dirección y Gestión de Centros Escolares.

juanfran.alvarez@ua.es 
Álvarez Herrero, J. F. y Roig-Vila, R. Las páginas web de los centros educativos. Análisis de la situación actual en la Comunidad Valenciana

\section{ABSTRACT}

The website has become an essential requirement of any educational center. Beyond being platforms for the information and communication of the entire educational community, today we find that these web pages are also shop windows of the centers. In them, the excellences of these centers are announced and shown and are a claim of educational marketing. Therefore it is essential to know if these letters of introduction of the centers have all the elements and characteristics that they must possess. This study analyzed 100 random web pages of non-university educational centers of the Valencian Community (Spain) and it was found that there are quite a few differences between the websites of public centers and those of concerted and private centers. In general, all of them presented occasional deficiencies, among which are: a lack of image and a suggestive aesthetic, lack of information, lack of updating, the non-use or dissemination of the center's social networks and the nondissemination or information of the teaching methodologies or the pedagogical style of the center. These problems are motivated by the lack of proper management and maintenance of the web pages, a function that often falls to the ICT coordinators of the center and rarely have time and training to perform this task. Likewise, it was detected that the websites of these educational centers rarely have a pedagogical purpose that encourages and promotes learning.

KEY WORDS: school websites - ICT - educational marketing - web content Coordinator ICT - educational centers - innovation.

\section{AS PÁGINAS WEB DOS CENTROS EDUCATIVOS. ANÁLISES DA SITUAÇÃO ATUAL NA COMUNIDADE VALENCIANA}

\section{RESUMO}

A página Web se converteu em um requisito imprescindível de qualquer centro educativo. Mais que ser uma plataforma para a informação e a comunicação de a comunidade educativa, na atualidade nos encontramos com que estas páginas web são também vitrines desses centros para a sociedade. Nelas, se anunciam e se mostram as excelências desses centros e são um reclamo de marketing educativo. Por isso, são imprescindíveis conhecer se estas cartas de apresentação dos centros têm todos os elementos e as características que devem possuir. Este estudo analisou 100 páginas web aleatoriamente de centros educativos nos universitários da Comunidade Valenciana (Espanha) e constatou que existem bastantes diferenças entre as webs de centros públicos e as de centros privados. Em geral, todas elas apresentavam alguma deficiência, entre as que se destacam são: falta de imagem e estética sugestiva, falta de informação, falta de atualização, o não uso e nem a difusão das redes sociais do centro e a falta de difusão ou informação das metodologias docentes ou o estilo pedagógico do centro. Esses problemas vêm motivados pela falta de uma correta gestão e manutenção das páginas webs, função que muitas vezes recaem nos coordenadores TIC do centro e que poucas vezes dispõem de tempo e formação para desempenhar estas tarefas. Mesmo assim, se 
Álvarez Herrero, J. F. y Roig-Vila, R. Las páginas web de los centros educativos. Análisis de la situación actual en la Comunidad Valenciana

detectou que as webs destes centros educativos poucas vezes têm uma finalidade pedagógica que incentive e promova a aprendizagem.

PALAVRAS CHAVE: páginas webs de centro educativo - TIC - marketing educativo - conteúdo web - coordenador TIC - centros educativos - inovação.

\section{Cómo citar el artículo:}

Álvarez Herrero, J. F. y Roig-Vila, R. (2019). Las páginas web de los centros educativos. Análisis de la situación actual en la Comunidad Valenciana. [The websites of schools. Analysis of the current situation in the Valencian Community]. Revista de Comunicación de la SEECI, (50), 129-147.

doi: http://doi.org/10.15198/seeci.2019.50.129-147

Recuperado de http://www.seeci.net/revista/index.php/seeci/article/view/623

\section{INTRODUCCIÓN}

La sociedad actual en la que nos encontramos inmersos vive volcada a Internet. Esta Red ha llegado a convertirse en un recurso sin el que muchas personas no podrían vivir. Además, con la incursión y el actual uso masivo de los dispositivos móviles, y más concretamente de los smartphones, vivimos permanentemente conectados. Todo ello ha supuesto una revolución del mundo tal y como lo conocemos y un cambio en nuestros hábitos, costumbres y actividades. Hoy en día compramos casi de todo en Internet y recurrimos menos a las tiendas físicas de toda la vida. Consumimos la información y el ocio a través de Internet y esto hace que medios como la prensa, la radio, la televisión, el cine o incluso el teatro, hayan tenido que reinventarse ante este cambio en el consumo de sus servicios.

Existen numerosos ejemplos y ámbitos donde Internet ha cambiado hábitos tradicionales. En educación no hemos sido ajenos a este cambio. Internet ha cambiado, no sólo la forma de enseñar y aprender, sino también de dar visibilidad a los centros educativos y lo que en ellos se hace, a publicitarse, a servir no sólo de plataforma de información y comunicación, sino también como el medio que posibilita la formación online e incluso la internacionalización de un centro educativo (Yemini y Cohen, 2016).

El sitio web, así pues, es un elemento que se ha convertido en imprescindible en cualquier centro educativo. Así por ejemplo, muchas familias al buscar un centro educativo para sus hijos, consultan en primer lugar el sitio web del centro educativo (Egido y Manero, 2012) y, en caso de no estar publicada esta información, inmediatamente quedará descartado.

Hoy en día buscamos estar permanentemente informados y deseamos conocer en todo momento lo que están haciendo nuestros hijos en el centro educativo (Gilleece y Eivers, 2018). El sitio web del centro se convierte en un elemento que permite visualizar todas las actividades, los proyectos y los progresos de nuestros hijos. A su vez, esta visualización sirve como elemento publicitario del centro hacia la sociedad. 
El sitio web del centro no es sólo, pues, una excelente herramienta o plataforma de información y comunicación (Marqués, 1998), sino que también sirve para publicitar y dar visibilidad al centro educativo (Dimopoulos y Tsami, 2018), así como ser un medio de formación, de gestión académica o incluso como repositorio de actividades y recursos.

A modo de resumen, cabe decir que las finalidades o funciones de un sitio web según Bedriñana (2005) son:

- Informativa: ofrecer información veraz sobre el centro educativo, sobre sus instalaciones, su estructura, sus funciones, sus actividades, servicios, etc.

- Comunicativa: servir de nexo de comunicación entre el centro y el resto de la comunidad educativa e incluso del mundo exterior.

- Formativa: ejerce la tarea de formar bien al alumnado, al profesorado, a las familias o incluso a otros agentes externos a la comunidad educativa.

- Repositorio de recursos, actividades, contenidos para el profesorado, alumnado, familias, etc.

- Otras funciones: orientadora, trabajo de clase, lúdica, etc.

Todas estas funciones u objetivos a cumplir por el sitio web de un centro educativo le confieren una gran importancia al mismo, lo cual implicaría que fuese objeto de múltiples investigaciones y estudios sobre su uso y funcionalidad en el mundo educativo, así como postularse como elemento básico y de suma importancia en todo centro educativo. Sin embargo, ni lo uno ni lo otro se da realmente. Por un lado, son muy pocos los estudios que se han realizado sobre los sitios webs de los centros educativos (Hartshorne, Friedman, Algozzine y Kaur, 2008; Poock, 2005; Tamatea, Hardy y Ninnes, 2008), aunque debemos citar el estudio que hicimos hace ya años, donde analizamos 1.115 páginas web escolares de las diecisiete comunidades autónomas españolas (Roig-Vila, 2003). También son pocos los estudios recientes (Álvarez-Álvarez, 2017; Gilleece y Eivers, 2018; Taddeo y Barnes, 2014; Tardío y Álvarez, 2018).

Por todo ello, nos planteamos como objetivo de esta investigación, realizar un análisis de la situación para conocer de una forma aproximada el estado actual de las webs de los centros educativos no universitarios de la Comunidad Valenciana.

Para realizar este análisis con rigor y calidad, consideramos que debemos profundizar, no solo en las funciones que pueda tener la página web y que ya hemos mencionado, sino también en las características y particularidades que hacen que estas webs sean tan singulares e importantes (López, 2007).

Por un lado, cabe destacar el hecho de si tienen una estructura organizada y una estética cuidada (Torres, 2005). Así mismo, en cuanto al contenido que esta ofrece, además de ser veraz y de calidad, debe contener toda aquella información pública que pueda necesitar la comunidad educativa. Como ejemplo cabe citar el proyecto educativo de centro, la programación general anual, los diferentes planes y proyectos de centro, etc. (Benito, Alegre y González, 2014). 
Por otro lado, debe ofrecer contenidos actualizados y enlaces que estén operativos, ser accesible a cualquier persona atendiendo a las posibles discapacidades que se puedan dar, estar ausente de publicidad y tener un carácter multilingüe, máxime cuando estamos hablando que en nuestra investigación vamos a analizar las webs de centros educativos de la Comunidad Valenciana, una comunidad que posee dos idiomas oficiales.

Es importante también tener muy presente a quién va dirigida la página web del centro educativo o, al menos, pensar en quién va a ser su audiencia mayoritaria. Lo correcto es hablar de webs educativas dirigidas a toda la comunidad educativa: alumnado, profesorado y familias, especialmente estas últimas.

Por último, cabe decir que existen aspectos importantes tales como que la web permita una fácil y rápida navegación, que cualquier contenido no esté más lejos de tres clics. Asimismo, ha de permitir la interacción con el usuario: no sólo debe ofrecerle los datos de contacto con una dirección física, una dirección de correo electrónico y un teléfono, sino que se deben abrir otros canales de comunicación e interacción con el usuario (McCabe y Weaver, 2018), como pueden ser las redes sociales, presentes prácticamente ya en todas las webs de los centros educativos (González, 2008; Roig-Vila, 2009). Por otro lado, no menos importante es dar difusión de la forma en que se trabaja en el centro educativo: qué metodologías o estilo educativo se utiliza en las aulas (Egido y Manero, 2012) y qué servicios se ofrecen, tanto los extraordinarios como los servicios básicos.

Somos conscientes de que llevar adelante una página web de un centro educativo con todas las funciones y características mencionadas, no está exento de dificultades y problemas. Un buen mantenimiento y seguimiento de la página web comporta disponer de una buena infraestructura con la que crearla y mantenerla. En la actualidad, en la Comunidad Valenciana se cuenta con la plataforma "Mestre a casa" que ofrece a los centros educativos públicos un sitio en Internet donde alojar su página web. Ya se ha anunciado su cambio a la plataforma WordPress tras años sin actualizaciones y un entorno obsoleto y limitado. Algunos centros públicos, así como otros tantos concertados y privados optan por opciones gratuitas como Google Sites o un blog de Blogger o WordPress, pero la mayoría de centros concertados y privados optan por entornos privados creados, y en ocasiones también gestionados, por empresas privadas.

A todo esto cabe sumarle la gestión de dichas páginas web respecto a quién se hace cargo de su continua actualización y producción de contenidos. En este sentido, hay bastante diversidad en este aspecto. Como ya hemos comentado, en algunos casos de centros privados y concertados hay empresas externas que se encargan de ello, mientras que en la mayoría de los casos, tanto en centros concertados, privados y en la totalidad de los públicos, se cuenta con la labor, bien del/de la coordinador/a TIC, bien de un equipo de docentes encargados de dicha gestión (Espuny, Gisbert, Coiduras y González, 2012; Rodríguez-Miranda, Pozuelos-Estrada y León-Jariego, 2014). Sea de un modo u otro, es un trabajo que, a excepción de cuando se encarga a una empresa privada, es de difícil gestión. En muchas ocasiones esta dificultad 
Álvarez Herrero, J. F. y Roig-Vila, R. Las páginas web de los centros educativos. Análisis de la situación actual en la Comunidad Valenciana

conlleva que la página web no esté actualizada o que su estética y contenido se muestren descuidados (Santos, García y Díaz, 2017).

Otros problemas derivados de otros condicionantes más variados hacen que la página web pueda presentar algunas deficiencias tales como: presencia de publicidad, ausencia de información relevante, como el proyecto educativo del centro o la programación general de aula, poco uso o ausencia total de las redes sociales, ausencia de aspectos que faciliten su accesibilidad a todas y todos (Wells y Barron, 2006), y que no ofrezca la posibilidad de ser consultada en otras lenguas.

Así, a partir de todo lo expuesto, nos propusimos llevar adelante esta investigación con el propósito de investigar las páginas web de centro y su importancia dentro de la eficacia y responsabilidad de un centro educativo, así como de los procesos de enseñanza-aprendizaje que de éste se derivan en un contexto concreto como es la Comunidad Valenciana.

\section{OBJETIVOS}

El objetivo principal de este estudio es conocer y analizar las páginas web de centros educativos no universitarios de la Comunidad Valenciana, pero este objetivo general lleva asociado, a su vez, otros objetivos secundarios a los que también pretendemos dar respuesta, como son:

- Conocer los principales problemas que presentan las páginas web de los centros educativos.

- Observar si se producen diferencias significativas entre las páginas web atendiendo a la titularidad de los centros.

- Analizar la principal utilidad o fin que se da a estas páginas web.

\section{METODOLOGÍA}

Dado que el ámbito de estudio es la Comunidad Valenciana y sus centros educativos no universitarios, para delimitar una muestra de estudio se tomaron los datos estadísticos de la Estadística de enseñanzas no universitarias de la Conselleria de Educación, Cultura y Deporte de la Comunidad Valenciana. Se tuvo en cuenta la titularidad de los centros, así como la proporcionalidad de 3 a 1 en los centros públicos entre los centros de educación infantil y primaria respecto a los centros de educación secundaria y bachillerato. Otra cuestión que se tuvo en cuenta fue la diferente proporcionalidad que existe en el número de centros educativos en las tres provincias de la Comunidad Valenciana. Atendiendo a una muestra de estudio de 100 páginas web, se distribuyó de forma homogénea y proporcional al número de centros que encontramos en la Comunidad Valenciana, tal y como se puede ver en la tabla 1. Así, se tuvo en cuenta los criterios de proporcionalidad comentados previamente, tanto en la titularidad del centro, como en los niveles de enseñanza que se imparten en los centros de titularidad pública y de su distribución geográfica. 
Álvarez Herrero, J. F. y Roig-Vila, R. Las páginas web de los centros educativos. Análisis de la situación actual en la Comunidad Valenciana

\section{Tabla 1. Centros educativos no universitarios de la Comunidad Valenciana y proporcionalidad sobre 100.}

\begin{tabular}{|l|c|c|c|c|c|}
\hline & C. Valenciana & $\%$ & & EI+EP & ESO+BAT \\
\hline $2017-18$ & 2.765 & & 100 & & \\
\hline Centros públicos & 1.702 & 61,555 & 62 & 41 & 21 \\
\hline Centros privados & 1.063 & 38,445 & & & \\
\hline Concertados & 412 & 14,901 & 15 & & \\
\hline No concertados & 651 & 23,544 & 23 & & \\
\hline
\end{tabular}

Fuente: Adaptado de Estadísticas de enseñanzas no universitarias.

La muestra de páginas web fue tomada al azar, sin ningún criterio especial, salvo los ya reseñados, con el fin de mantener la proporcionalidad en cuanto a la titularidad, etapas educativas y provincia de los centros.

Como instrumento de análisis, se ha elaborado uno ad hoc resultante de tomar en consideración las dimensiones y variables tomadas por Álvarez-Álvarez (2017) en su estudio, así como algunos de los campos considerados en la propuesta de Marqués (1999) y la nuestra propia (Roig-Vila, 2003).

Se analizaron 6 dimensiones: 1.- Identificación, 2.- Presentación y organización de la información, 3.- Contenido, 4.- Audiencia, 5.- Navegación y 6.- Metodologías docentes y servicios. Todas ellas, a su vez, contemplan una serie de variables que sólo ofrecen la posibilidad de cumplimiento o incumplimiento de las mismas, aunque en algunos casos más particulares hay opciones cerradas y limitadas a 3 o 4 posibles respuestas. Dichas dimensiones, con sus variables y definición, se pueden consultar en la tabla 2.

Dado que la gran mayoría de las variables presentaban esta dicotomía de respuesta y en las restantes también un número cerrado y limitado de posibles respuestas, se realizó un análisis estadístico descriptivo de contabilización de frecuencias y que en los resultados aparecerán traducidas en porcentajes para un mejor análisis de los mismos.

Tabla 2. Dimensiones, variables y su definición.

\begin{tabular}{|l|c|c|}
\hline \multicolumn{1}{|c|}{ Dimensión } & Variables & Definición \\
\hline \multirow{2}{*}{ 1.- Identificación } & Titularidad & $\begin{array}{r}\text { Público: EI+EP / ESO+Bat+FP } \\
\text { Concertado } \\
\text { Privado }\end{array}$ \\
\cline { 2 - 3 } & Autoría & Presenta la autoría de la web: sí/no \\
\cline { 2 - 3 } & Licencias & Presenta las licencias de uso de la web: sí/no \\
\hline \multirow{2}{*}{$\begin{array}{l}\text { 2.- Presentación } \\
\text { y organización de } \\
\text { la información }\end{array}$} & Portal & Mestre a casa \\
& & $\begin{array}{c}\text { Blog/Google Sites/Wix } \\
\text { Portal privado }\end{array}$ \\
\cline { 2 - 3 } & Imagen & Estética cuidada: sí/no \\
\hline
\end{tabular}


Álvarez Herrero, J. F. y Roig-Vila, R. Las páginas web de los centros educativos. Análisis de la situación actual en la Comunidad Valenciana

\begin{tabular}{|c|c|c|}
\hline & Organización & Responde a un diseño organizativo previo: sí/no \\
\hline \multirow[t]{8}{*}{ 3.- Contenido } & Información & Es una web nutrida de informaciones: sí/no \\
\hline & $\begin{array}{l}\text { Documentos } \\
\text { institucionales y } \\
\text { Planes de centro }\end{array}$ & $\begin{array}{c}\text { Es posible conocer los documentos } \\
\text { institucionales (proyecto educativo, } \\
\text { programación general anual, plan de atención a } \\
\text { la diversidad, etc.): sí/no }\end{array}$ \\
\hline & Actualización & $\begin{array}{l}\text { Actualizada: semanal, mensual, trimestral, } \\
\text { semestral, un año o más }\end{array}$ \\
\hline & $\begin{array}{l}\text { Soporte de contenido } \\
\text { predominante }\end{array}$ & $\begin{array}{c}\text { Texto: sí/no } \\
\text { Imagen: sí/no } \\
\text { Vídeo: sí/no }\end{array}$ \\
\hline & Accesibilidad & Es accesible a personas con discapacidad: sí/no \\
\hline & Links externos & Los enlaces externos están actualizados: sí/no \\
\hline & Publicidad & Ausencia de publicidad: sí/no \\
\hline & Multilingüe & Presenta carácter multilingüe: sí/no \\
\hline 4.- Audiencia & $\begin{array}{l}\text { Público a quien va } \\
\text { dirigida la web }\end{array}$ & $\begin{array}{c}\text { Profesorado: sí/no } \\
\text { Alumnado: sí/no } \\
\text { Familias: sí/no }\end{array}$ \\
\hline \multirow{3}{*}{$\begin{array}{l}\text { 5.- Navegación, } \\
\text { Interacción y/o } \\
\text { Comunicación }\end{array}$} & Navegación & $\begin{array}{l}\text { La navegación por la web es fácil, rápida y se } \\
\text { gestiona en } 3 \text { o menos enlaces: sí/no }\end{array}$ \\
\hline & Interacción & $\begin{array}{l}\text { La web permite la interacción con el usuario: } \\
\text { sí/no }\end{array}$ \\
\hline & Redes Sociales & $\begin{array}{l}\text { La web facilita el conocimiento y/o seguimiento } \\
\text { de las redes sociales del centro: sí/no }\end{array}$ \\
\hline \multirow[t]{2}{*}{$\begin{array}{l}\text { 6.- Metodologías } \\
\text { docentes y } \\
\text { servicios }\end{array}$} & $\begin{array}{l}\text { Metodologías } \\
\text { docentes o estilo } \\
\text { educativo }\end{array}$ & Es posible conocerlas: sí/no \\
\hline & Otros servicios & $\begin{array}{l}\text { Permite conocer otros servicios que ofrezca el } \\
\text { centro (comedor, extraescolares, AMPA, etc.): } \\
\text { sí/no }\end{array}$ \\
\hline
\end{tabular}

Fuente: Elaboración propia.

Para contrastar y triangular las respuestas obtenidas de este análisis, se pasó el mismo instrumento a 33 coordinadores TIC o responsables del mantenimiento y gestión de las correspondientes páginas web del total de 100 de la muestra y se constató la fiabilidad y la coincidencia de las respuestas obtenidas con las del estudio. El análisis se realizó durante el mes de febrero de 2019.

\section{RESULTADOS}

En la actualidad todos los centros educativos de enseñanzas no universitarias de la Comunidad Valenciana cuentan con un espacio web en Internet por el que se hacen visibles. Ahora bien, como veremos a continuación, existen diferencias notables entre los diversos centros y sus portales o webs, así como deficiencias y errores de uso bastante generalizados. A continuación, pasamos a analizar los resultados obtenidos de este estudio descriptivo atendiendo a las 6 dimensiones estudiadas: 1.Identificación, 2.- Presentación y organización de la información, 3.- Contenido, 4.Audiencia, 5.- Navegación y 6.- Metodologías docentes y servicios.

Revista de Comunicación de la SEECI. 15 noviembre, 2019 / 15 marzo, 2020, nº 50, 129-147 
Álvarez Herrero, J. F. y Roig-Vila, R. Las páginas web de los centros educativos. Análisis de la situación actual en la Comunidad Valenciana

\subsection{Identificación}

Tal y como ya se ha comentado, atendiendo a la diferente titularidad que presentan los centros educativos de enseñanzas no universitarias de la Comunidad Valenciana, en nuestra muestra de 100 centros, se tomaron 62 centros públicos (41 de Educación Infantil y Educación Primaria, y 21 de Educación Secundaria y Ciclos de Formación Profesional), 15 centros concertados integrados (con niveles de enseñanza que van desde la Ed. Infantil a la Ed. Secundaria) y 23 centros privados.

La autoría de estas páginas web muy pocas veces se ve reflejada. Así en el caso de los centros públicos, al disponer de un portal como "Mestre a casa" ofrecido por la propia Conselleria de Educación, ya en su definición establece que los entornos webs proporcionados por "Mestre a casa" son diseñados, creados y mantenidos por los mismos usuarios del portal, sin que quede definida de una forma más precisa su autoría.

En el caso de centros privados y en la gran mayoría de los centros concertados, las páginas web han estado realizadas por empresas privadas y así se refleja en las mismas, aunque el mantenimiento y la gestión, como en el caso de las públicas, se lleve a cabo por docentes o el/la coordinador/a TIC del centro. Solo en dos casos no se encontró referencia alguna a la autoría de la página web del centro, ni al nombre de una empresa, ni tampoco a nombre de docentes del centro.

En cuanto a las licencias de uso de las diferentes páginas web, nos encontramos con iguales resultados a lo que veíamos antes con la autoría. Tanto las páginas webs dependientes del portal "Mestre a casa", como las pertenecientes a empresas 0 entidades privadas, están sujetas a Copyright y sólo en aquellos casos en los que se hace un uso de blogs o recursos gratuitos disponibles en la Red, se hace uso de otro tipo de licencias, que obedecen en la mayoría de los casos a licencias Creative Commons. Sólo en 4 casos presentaban este tipo de licencias Creative Commons, mientras que las 96 restantes presentaban Copyright.

\subsection{Presentación y organización de la información}

Como ya se ha comentado, los centros de titularidad pública cuentan con un espacio web asignado en la plataforma "Mestre a casa", y es aquí, por tanto, donde se encuentran alojadas las páginas web de los centros públicos. Sin embargo, entre las 62 páginas web de centros públicos testeadas en esta investigación, se han encontrado dos centros públicos que han recurrido a otras opciones (un blog de WordPress y un sites de Google respectivamente) para albergar sus páginas web de forma paralela a sus espacios (abandonados o sin desarrollar) en "Mestre a casa".

En total, sin atender a la titularidad de los centros, encontramos que el $60 \%$ hace uso de la plataforma "Mestre a casa", un 36\% usa un portal privado y sólo un $4 \%$ hace uso de algún recurso o herramienta de índole gratuita como son blogs de WordPress y Blogger, o el Sites de Google (ver gráfico 1).

Revista de Comunicación de la SEECI. 15 noviembre, 2019 / 15 marzo, 2020, nº 50, 129-147 
Álvarez Herrero, J. F. y Roig-Vila, R. Las páginas web de los centros educativos. Análisis de la situación actual en la Comunidad Valenciana

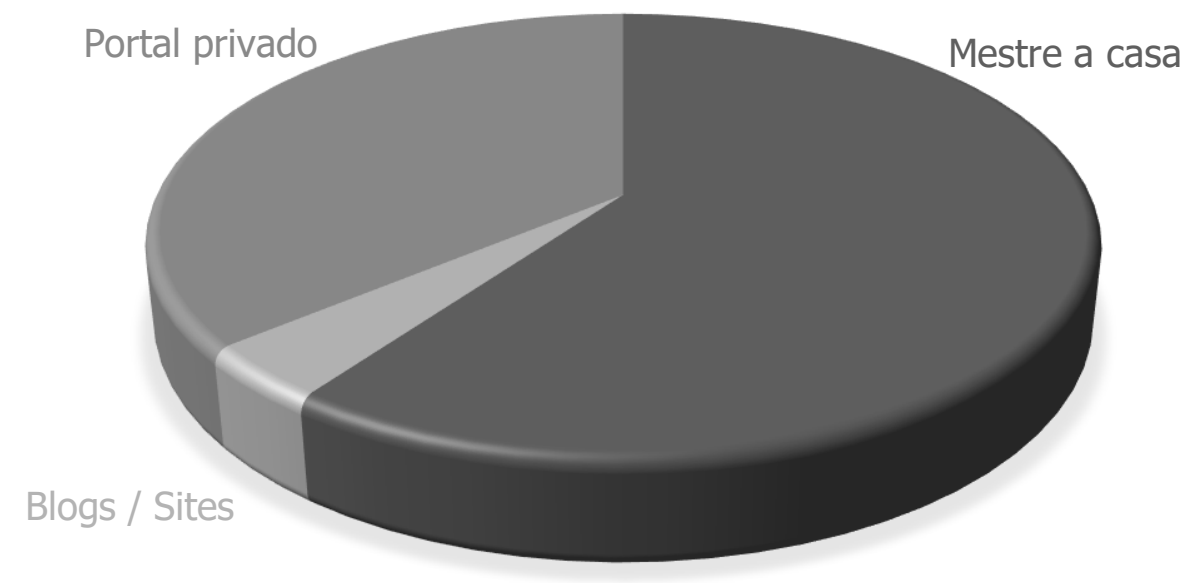

Gráfico 1: Portal donde se alberga la correspondiente página web.

Fuente: Elaboración propia.

Atendiendo a la imagen, la estética y el diseño de la página web, solamente hemos tenido en cuenta un criterio muy general en el que se ha considerado si el cuidado y la estética del lugar invita o no a su visita, y si el aspecto está cuidado o, por el contrario, ofrece cierta sensación de abandono y dejadez. Encontramos que tan sólo 54 de las webs ofrecen un sensación agradable, con mensaje de bienvenida, con imágenes sugerentes y tamaño y estilo de letra adecuado.

El $46 \%$ de las páginas restantes dan una sensación de abandono, de no cuidar los aspectos estéticos en cuanto a presentación, imagen y texto idóneos. Sí que se constata una diferencia importante atendiendo a la titularidad de los centros, pues de las 46 páginas que presentan una estética deficiente, 43 de ellas pertenecen a centros de titularidad pública. En su defensa hay que argumentar que el portal "Mestre a casa" ofrece pocas posibilidades de personalización y "customización" y, al poseer una estructura bastante rígida, limita mucho poder crear un entorno amigable y atractivo.

Respecto a la organización, sí se da un elevado porcentaje de páginas web bien organizadas y estructuradas. Disponen de menús y botones que facilitan la clasificación de la información que en ellas se dispone. Solo en 3 de los casos analizados, bien por la ausencia de una estructura o por una caótica presencia de la misma, podemos decir que la organización de la página web no responde a un diseño organizativo previo.

\subsection{Contenido}

La información que debe aportar una página web es uno de los pilares básicos de la misma para cualquier usuario que accede a ella. Así pues, la información debe ser 
nutrida, variada y actualizada. En cuanto a la cantidad de información, encontramos que en 43 de los casos analizados, esta es más bien nula o escasa. Este porcentaje es preocupante, y más en nuestros días donde Internet es crucial en nuestra sociedad y es la principal fuente de información del ciudadano actual. Asimismo, vuelve a ser preocupante que de estos 43 casos, la gran mayoría de ellos corresponden a páginas web de centros públicos (38), en los que en sus webs de "Mestre a casa" sólo ofrecen la información básica establecida al inicio de cada curso escolar por la propia Conselleria y poco más.

Igualmente ocurre con la documentación oficial. Tal y como se establece en la LOMCE en su artículo 121.3 y 121.6 y la orden EDU/25/2010, los proyectos educativos de los centros deben de ser públicos. En este sentido, encontramos que en 56 de los casos analizados no se encuentra disponible dicha información, así como tampoco otros planes: de convivencia, TIC, lector, atención a la diversidad, etc. En este caso, sí se encuentra más repartida la responsabilidad de hacer pública esta información: de los 56 casos detectados, 37 corresponden a centros públicos, 8 a centros concertados y 11 a centros privados.

Preocupante resulta la gran diferencia existente entre centros públicos y concertados y privados atendiendo a la actualización de los contenidos. En centros públicos sólo 22 de los 62 , es decir un 35,5\%, mantienen una página web actualizada con una frecuencia como mínimo trimestral y, por el contrario, en centros concertados y privados los porcentajes de páginas web actualizadas con una frecuencia como mínimo mensual supera el $90 \%$ en ambos casos tal y como se puede apreciar en la tabla 3.

Tabla 3. Frecuencia de actualización de la página web atendiendo a la titularidad del centro.

\begin{tabular}{|c|c|c|c|c|c|c|c|c|c|c|}
\hline \multirow{2}{*}{ Titularidad } & \multicolumn{2}{|c|}{ Semanal } & \multicolumn{2}{|c|}{ Mensual } & \multicolumn{2}{|c|}{ Trimestral } & \multicolumn{2}{|c|}{ Semestral } & \multicolumn{2}{|c|}{ Anual o + } \\
\hline & $\mathbf{f}$ & $\%$ & $\mathbf{f}$ & $\%$ & $\mathbf{f}$ & $\%$ & $\mathbf{f}$ & $\%$ & $\mathbf{f}$ & $\%$ \\
\hline Público & 6 & 9,7 & 8 & 12,9 & 8 & 12,9 & 35 & 56,5 & 5 & 8,0 \\
\hline Concertado & 12 & 80 & 2 & 13,3 & 0 & 0 & 1 & 6,7 & 0 & 0 \\
\hline Privado & 18 & 78,3 & 4 & 17,4 & 1 & 4,3 & 0 & 0 & 0 & 0 \\
\hline
\end{tabular}

Fuente: Elaboración propia.

El soporte de contenido predominante en la página principal o de inicio de la página web también presenta diferencias bastante considerables entre centros públicos y privados, pero ello se debe, principalmente, a las pocas posibilidades que la plataforma "Mestre a casa" ofrece a las páginas web de los centros públicos. Si bien posibilita la inserción, tanto de imágenes como de vídeos, su dificultad de inserción, su baja calidad, la dependencia de otros recursos abiertos como repositorios de vídeos públicos, el cumplimiento de la ley de protección de datos del alumnado, etc., hacen que en muchos casos se desestime su uso. Así, en las páginas web de los centros públicos, en 44 de ellas (71\%) predomina el formato texto frente al audiovisual. En cambio, en los centros concertados predomina la imagen en 14 
Álvarez Herrero, J. F. y Roig-Vila, R. Las páginas web de los centros educativos. Análisis de la situación actual en la Comunidad Valenciana

páginas de $15(93,3 \%)$ y en los privados la imagen está presente en la totalidad de ellas $(23,100 \%)$.

Resulta bastante preocupante la poca atención a la accesibilidad de las páginas web de los centros escolares, pues en ninguno de los 100 casos analizados pudimos encontrar ningún tipo de recurso o facilidad para la navegación sin barreras en aquellas personas con algún tipo de discapacidad.

La actualización de los enlaces externos que ofrecen las páginas web es bastante notable, al menos en los centros de titularidad concertada y privada, ya que no se encontró ningún caso de enlaces caducados. Sin embargo, no es el caso de los centros públicos, donde en 8 de las 62 webs $(12,9 \%)$ de centros públicos analizadas se encontraron enlaces no operativos.

Respecto a la posibilidad de encontrar publicidad en las webs, las cifras pesan en contra de los centros de carácter concertado (en 4 de las 15 se encontró publicidad directa o indirecta, un $26,7 \%$ ) y de carácter privado (en 7 de las 23 analizadas se encontró publicidad, un $30,4 \%$ ), frente a ninguna página en el caso de los centros públicos.

Respecto al carácter multilingüe de las páginas web, hay que realizar algunas consideraciones previas, pues si bien los centros públicos a través de "Mestre a casa" ofrecen la posibilidad de un carácter bilingüe (valenciano y castellano), éste no es del todo real, pues sólo se limita a lo que es la estructura de la web -la traducción de los títulos de los menús, de los apartados y subapartados que presenta la web-, pero no al contenido, que está escrito únicamente en una de las dos lenguas. Lo mismo podemos decir en el caso de las páginas web de la mayoría de los centros concertados y privados que ofrecen esta posibilidad: cuando se accede a los contenidos, estos son en un idioma u otro. Solo hay que reseñar un caso de todos los analizados en el que sí se presenta un bilingüismo completo y que corresponde a un centro privado de marcado carácter lingüista francés y que presenta todos los contenidos de la web en castellano y francés.

\subsection{Audiencia}

Como ya hemos mencionado en la introducción de este estudio, toda web educativa debe ser apoyo y herramienta útil para la comunidad educativa y esto incluye, al menos, los tres colectivos principales de ésta: alumnado, profesorado y familias. Cuando pasamos a analizar la audiencia a quien van dirigidas las 100 páginas web analizadas, nos encontramos que en un $100 \%$ sí se muestran enfocadas a informar y ayudar en la difusión y conocimiento del centro a las familias.

Respecto al profesorado, en ocasiones puede tener su espacio en dichas webs y así se constata en 89 de ellas, aunque hay que hacer la puntualización de que se han considerado la totalidad de páginas web sustentadas en "Mestre a casa". En éstas, se ofrece la posibilidad al profesorado del centro de enlazar con sus blogs o espacios 
Álvarez Herrero, J. F. y Roig-Vila, R. Las páginas web de los centros educativos. Análisis de la situación actual en la Comunidad Valenciana

dentro de la misma plataforma, aunque en muchos casos estos espacios no se usan o dejaron de usarse hace mucho tiempo.

Con todo ello, sólo en 4 webs de centros concertados y en 7 de centros privados, no se ofrece ningún contenido, ni posibilidad de uso o utilidad para el profesorado. Por otro lado, sí resulta muy preocupante el hecho de que no se dé opción al alumnado de hacer un uso útil y productivo de la web del centro como un recurso y/o herramienta más de su proceso de enseñanza-aprendizaje. En ninguna web de los centros públicos se da esta opción y solo se da en el caso de 3 de los concertados y en 8 de los privados.

\subsection{Navegación}

La navegación a través de una página web tiene que ser ágil, rápida y directa. Por ello, en una página web debemos poder llegar a encontrar o acceder a aquella información o contenido que buscamos en menos de tres clics de ratón. Afortunadamente, en todos los casos analizados se cumple esta regla de los tres clics. Sin embargo, sí se ha constatado algún caso en el que la posibilidad de interacción y comunicación entre los diversos agentes de la comunidad educativa o entre el/la navegante y el centro, no se produce.

Más allá de la comunicación que se puede establecer ofreciendo los datos de contacto, como son la dirección física del centro, el teléfono y el email, está el ofrecer algún otro tipo de recurso o medio con el que navegantes y centro puedan estar comunicados. Si bien en la totalidad de los casos analizados se facilitan estos datos comentados (dirección, teléfono y email), cuando hablamos de otro tipo de comunicación e interacción como chats, encuestas de opinión, formularios de participación, posibilidad de realizar comentarios, etc., tan sólo se da en 12 webs ( 5 de centros concertados y 7 de centros privados).

Caso aparte merece el análisis del uso de las redes sociales y su difusión en la web del centro, pues si bien se da en la totalidad de los centros concertados y privados, no ocurre lo mismo en los centros públicos, donde sólo se da en 19 de los 62 centros (30,6\%). Así, se observa un amplio uso de Facebook así como un creciente uso de Instagram frente a otras redes sociales que experimentan cierto retroceso en su uso y difusión, como es el ejemplo de Twitter.

\subsection{Metodologías docentes y servicios}

Es muy importante que el uso de la web del centro vaya más allá de ser un escaparate en el que mostrar a las familias las excelencias del centro para que se considere como el candidato perfecto ante la posible elección de un centro escolar para sus hijos. Sin embargo, no podemos olvidar que una de las primeras cosas que hacen las familias que buscan un centro docente para sus hijos es buscar la web de los centros candidatos.

Cuando se accede a la misma, se buscan los servicios que ofrece y las metodologías docentes o estilo educativo que utilizan. En el análisis de estos dos 
aspectos nos hemos encontrado un desigual resultado, pues si bien el $100 \%$ de las webs hacen mención a los servicios que ofrecen (comedor escolar, extraescolares, transporte escolar, AMPA, etc.), no ocurre lo mismo con la difusión o información del estilo educativo del centro o las metodologías educativas que se utilizan. Nuevamente encontramos una gran diferencia entre los centros públicos y los concertados y privados. En estos últimos, prácticamente en la totalidad de ellos (en 14 de 15 de los concertados, y en 22 de 23 de los privados) se hace mención, o incluso alarde, de las metodologías usadas en su modelo o estilo de aprendizaje. En cambio, en los centros públicos sólo se da en 18 de las 62 páginas analizadas (29\%).

A modo de conclusión, en el gráfico 2 se muestran aquellas variables en las que las diferencias entre centros públicos y concertados y privados son más relevantes.

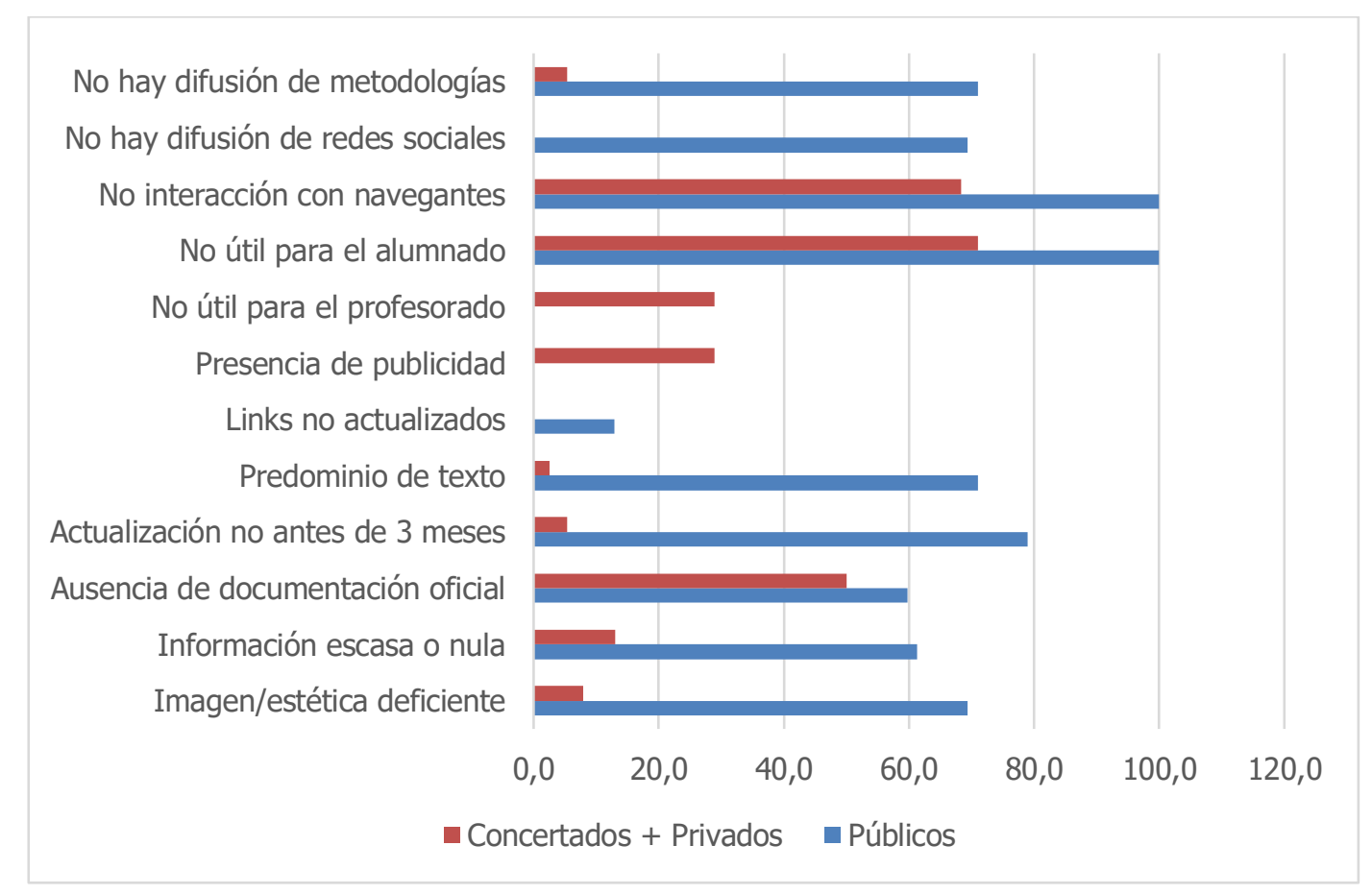

Gráfico 2: Diferencias en los porcentajes de diferentes variables entre centros públicos y concertados y privados.

Fuente: Elaboración propia.

\section{DISCUSIÓN}

A tenor de los resultados obtenidos en esta investigación, se comprueba que las características de las páginas web de los centros educativos de enseñanzas no universitarias de la Comunidad Valenciana es bastante variopinta. Aun así, se pueden establecer algunas conclusiones, plantear nuevas hipótesis y futuras líneas de investigación en este campo de investigación.

A nivel general, cabe decir que, al comprobar que la totalidad de los centros escogidos al azar de entre la totalidad de centros educativos de ámbito no universitario de la Comunidad Valenciana cuenten con página web, es representativo 
Álvarez Herrero, J. F. y Roig-Vila, R. Las páginas web de los centros educativos. Análisis de la situación actual en la Comunidad Valenciana

en el sentido de la existencia generalizada de página web en cualquier centro educativo.

Ahora bien, este resultado tan óptimo no podemos aplicarlo a los tres objetivos específicos que nos planteábamos al principio. Así, atendiendo al primer objetivo, respecto a los principales problemas que presentan las páginas web, no podemos dejar de lado el segundo objetivo, pues, como hemos visto, sí existen marcadas diferencias entre las webs de centros públicos y las de centros concertados y privados. Así, las webs de centros públicos presentan como principales problemas una falta de imagen y una estética atractiva en sus webs, la falta de información, la falta de actualización, el predominio de texto frente al recurso audiovisual, el no uso ni difusión de las redes sociales del centro y la no difusión o información de las metodologías docentes o el estilo pedagógico del centro.

Por el contrario, los centros concertados presentan otro tipo de problemas como son la presencia de publicidad en sus webs, o el no ser útil para el profesorado. Como problemas comunes a los tres tipos de titularidad de centros, encontramos la falta de documentación oficial, el no ser de utilidad para el alumnado o el no ofrecer más interacción con los navegantes que la estrictamente necesaria (la dirección física del centro, el teléfono y el mail de contacto).

Como vemos, se puede constatar, no solo un gran número de problemas, sino también una clara diferencia entre las webs de centros públicos y las de centros concertados y privados. En la mayoría de los casos se trata de problemas corregibles y que, quizás, una nueva plataforma que sustituya a "Mestre a casa" permita solucionar muchas de estas deficiencias, así como reducir las diferencias entre los centros públicos y los concertados y privados.

Cabe señalar también un problema presente en los centros y que han constatado 33 de los 100 coordinadores TIC entrevistados como es la gestión y mantenimiento de las páginas web. Dichos coordinadores manifiestan la dificultad que supone gestionar y mantener las webs de los centros educativos. Las principales dificultades de las que se hacen eco son la falta de horas para su gestión, así como la dificultad y poca preparación que se cuenta para ello (no debemos olvidar que estamos hablando de docentes que no están preparados para dichas tareas). Mientras esta cuestión no se solucione, estos problemas a los que hacíamos mención, no desaparecerán. Además, esta cuestión parece ser más complicada de solucionar entre los centros públicos que entre los centros concertados y privados, por no poder disponer de empresas privadas que se encarguen de sus tareas.

Por último, en lo referente al tercer objetivo, referido a conocer la utilidad o fin que se le confiere desde los centros educativos a sus páginas web, hemos podido comprobar dos aspectos importantes. Por un lado, cabe decir que se conciben y mantienen las webs de los centros educativos como escaparates o medios para la difusión de las excelencias que presentan. Esto conlleva al segundo aspecto y es que las webs no se contemplan como medios o recursos que puedan servir para el fin principal y último de la educación como es permitir, favorecer y fortalecer los 
Álvarez Herrero, J. F. y Roig-Vila, R. Las páginas web de los centros educativos. Análisis de la situación actual en la Comunidad Valenciana

procesos de enseñanza-aprendizaje del alumnado, principalmente, pero también del resto de la comunidad educativa. Este último aspecto lo consideramos acuciante, pues se corre el peligro de que las páginas web de centros educativos acaben siendo únicamente elementos de márquetin educativo.

Para finalizar, cabe indicar que somos conscientes de las limitaciones de este estudio, derivadas de aspectos sobre el posible sesgo del evaluador, la muestra, etc., pero consideremos que, debido a las pocas investigaciones sobre este tema, resulta interesante como una primera toma de contacto y análisis de la realidad actual de las páginas web educativas de la Comunidad Valenciana. Es así como nos fijamos como meta seguir investigando dicho campo y ampliar el estudio a una muestra mayor y representativa que nos dé datos más fiables de la realidad que, como ya hemos visto, se antoja compleja y preocupante. En este mismo sentido, consideremos interesante establecer, como posibles líneas futuras de investigación, la propuesta de un plan de formación en la gestión y mantenimiento de webs educativas y de concienciación y usabilidad como medios y recursos para fomentar e incentivar desde las webs de los centros educativos el proceso de enseñanza-aprendizaje de toda la comunidad educativa.

\section{REFERENCIAS}

Álvarez-Álvarez, C. (2017). ¿Qué me ofrecen las Páginas Web de los Centros Educativos? Estudio Exploratorio en Cantabria (España). REICE, Revista Iberoamericana sobre Calidad, Eficacia y Cambio en Educación, 15(3), 49-63. doi: http://dx.doi.org/10.15366/reice2017.15.3.003

Bedriñana Ascarza, A. (2005). Técnicas e indicadores para la evaluación de portales educativos en Internet. Gestión en el Tercer Milenio, Revista de Investigación de la Facultad de Ciencias Administrativas, 14(7), 81-87.

Benito, R.; Alegre, M. Á. y González, I. (2014). School educational project as a criterion of school choice: discourses and practices in the city of Barcelona. Journal of Education Policy, 29(3), 397-420. doi: http://dx.doi.org/10.1080/02680939.2013.844858

Dimopoulos, K. \& Tsami, M. (2018). Greek Primary School Websites: The Construction of Institutional Identities in a Highly Centralized System,. Leadership and Policy in Schools, 174), 397-421. doi: http://dx.doi.org/10.1080/15700763.2017.1326147

Egido, M. P. y Manero, P. V. (2012). Libertad de elección, competencia y calidad: las políticas educativas de la Comunidad de Madrid. Profesorado. Revista de currículum y formación de profesorado, 16(3), 149-166.

Espuny, C.; Gisbert, M.; Coiduras, J. y González, J. (2012). El coordinador TIC en los centros educativos: funciones para la dinamización e incorporación didáctica de las 
Álvarez Herrero, J. F. y Roig-Vila, R. Las páginas web de los centros educativos. Análisis de la situación actual en la Comunidad Valenciana

TIC en las actividades de aprendizaje. Pixel-bit. Revista de Medios y Educación, (41), 7-18.

Gilleece, L. \& Eivers, E. (2018). Primary school websites in Ireland: how are they used to inform and involve parents? Irish Educational Studies, 374), 411-430. doi: http://dx.doi.org/0.1080/03323315.2018.1498366

González, F. S. (2008). Redes sociales y comunidades educativas. TELOS 76: Redes Sociales, (76), 99-109.

Hartshorne, R.; Friedman, A.; Algozzine, B. \& Kaur, D. (2008). Analysis of Elementary School Web Sites. Journal of Educational Technology \& Society, 11(1), 291-303.

Lopéz Carreño, R. (2007). Los portales educativos: clasificación y componentes, en Anales de documentación, (10), 233-244.

Marqués, P. (1998). Usos educativos de Internet: ¿̇la revolución en la enseñanza?, en Comunicación y Pedagogía, (154), 37-44.

Marqués, P. (1999). Criterios para la clasificación y evaluación de espacios web de interés educativo. Educar, (25), 95-111.

McCabe, M. B. \& Weaver, R. (2018). Marketing Effectiveness of Educational Services on Websites. Business Education \& Accreditation, 10(1), 49-58.

Poock, M. (2005). Determining the design of effective graduate school web sites. College and University Journal, 80(3), 23-26.

Rodríguez-Miranda, F. P.; Pozuelos-Estrada, F. J. y León-Jariego, J. C. (2014). The role of ICT coordinator. Priority and time dedicated to professional functions. Computers \& Education, (72), 262-270. doi: http://dx.doi.org/10.1016/j.compedu.2013.11.009

Roig-Vila, R. (2003). Análisis y valoración de sitios web de centros escolares. New York: The Edwin Mellen Press.

Roig Vila, R. (2009). Redes sociales y comunidades virtuales en la Web 2.0: implicaciones en el ámbito educativo. En R. Roig (coord.), Investigar desde un contexto educativo innovador (399-412). Alcoi: Marfil.

Santos, A. M.; García, J. A. C. \& Díaz, R. G. (2017). The websites of primary and secondary schools in Portugal: an evaluation proposal, en Education in the Knowledge Society, 18(3), 37-58. doi: http://dx.doi.org/10.14201/eks20171833758 
Álvarez Herrero, J. F. y Roig-Vila, R. Las páginas web de los centros educativos. Análisis de la situación actual en la Comunidad Valenciana

Taddeo, C. \& Barnes, A. (2014). The school website: Facilitating communication engagement and learning. British Journal of Educational Technology, 47(2), 421436. doi: http://dx.doi.org/10.1111/bjet.12229

Tamatea, L.; Hardy, J. \& Ninnes, P. (2008). Paradoxical inscriptions of global subjects: Critical discourse analysis of international schools' websites in the AsiaPacific Region, en Critical Studies in Education, 49(2), 157-170. doi: http://dx.doi.org/10.1080/17508480802040241

Tardío, V. y Álvarez, C. (2018). Análisis de las Páginas Web de los Centros Públicos de Educación Secundaria de Cantabria (España). REICE, Revista Electrónica Iberoamericana sobre Calidad, Eficacia y Cambio en Educación, 16(3), 49-65.

Torres Barzabal, L. M. (2005). Elementos que deben contener las páginas WEB educativas. Pixel-Bit. Revista de Medios y Educación, (25), 75-83.

Wells, J. A. \& Barron, A. E. (2006). School web sites: Are they accessible to all? Journal of Special Education Technology, 21(3), 23-30. doi: http://dx.doi.org/10.1177/016264340602100303

Yemini, M. \& Cohen, A. School websites as a novel internationalization assessment tool. Education and Information Technologies, 21(3), 607-623. doi: http://dx.doi.org/10.1007/s10639-014-9343-7

\section{AUTORES:}

\section{Juan Francisco Álvarez Herrero}

Doctor en Tecnología Educativa por la Universitat Rovira i Virgili. Profesor asociado del Departamento de Didáctica General y Didácticas Específicas de la Universidad de Alicante y miembro del grupo de investigación: EDUTIC-ADEI (Educación y Tecnologías de la Información y Comunicación-Atención a la Diversidad. Escuela Inclusiva) de la misma universidad. Su investigación se interesa especialmente por la implementación de pedagogías emergentes y metodologías activas, la competencia digital, los entornos personales de aprendizaje y las redes sociales en el mundo educativo.

juanfran.alvarez@ua.es

Orcid ID: http://orcid.org/0000-0002-9988-8286

Google Scholar: https://scholar.google.es/citations?hl=es\&user=DbUP2SkAAAAJ

ResearchGate: https://www.researchgate.net/profile/Juan Francisco Alvarez

Academia.edu: https://alicante.academia.edu/JuanFcoAlvarez

ResearcherID: http://www.researcherid.com/rid/L-2850-2017

\section{Rosabel Roig-Vila}

Doctora en Pedagogía y profesora titular de la Universidad de Alicante del Área de Didáctica y Organización Escolar. Ha sido decana de la Facultad de Educación y actualmente es la directora del Instituto de Ciencias de la Educación en esta universidad. Es directora de la Journal of New Approaches in Educational Research y 
Álvarez Herrero, J. F. y Roig-Vila, R. Las páginas web de los centros educativos. Análisis de la situación actual en la Comunidad Valenciana

coordinadora del grupo de investigación EDUTIC-ADEI (Educación y TIC-Atención a la Diversidad. Escuela Inclusiva). Su investigación se centra en la articulación de las TIC en Educación.

rosabel.roig@ua.es

Orcid ID: http://orcid.org/0000-0002-9731-430X

Google Scholar: https://scholar.google.es/citations?user=VSec3AkA

ResearchGate: https://www.researchgate.net/profile/Rosabel Roig-Vila

Academia.edu: https://alicante.academia.edu/rosabelroigvila

ResearcherID: http://www.researcherid.com/rid/D-5586-2014 